\title{
Three Highly Stable Cobalt MOFs Based on "Y"-shaped Carboxylic Acid: Synthesis and Absorption of Anionic Dyes
}

Wei Yan, Li-Juan Han, Hai-Lang Jia, Kang Shen, Ting Wang, and He-Gen Zheng*

State Key Laboratory of Coordination Chemistry, School of Chemistry and Chemical Engineering, Collaborative Innovation Center of Advanced Microstructures, Nanjing University, Nanjing 210023, P. R. China

\section{Contents}

\section{Experimental section.}

\section{Powder X-ray diffraction patterns of complexes 1-3.}

\section{Photographic images}

4. IR spectra of complexes 1-3 and related ligands.

5. The TGA diagrams of complexes 1-3.

6. The UV-visible spectra of complexes 1-3.

\section{Experimental section.}

Materials and measurements. Reagents and solvents employed were commercially available. Ligand $\mathrm{H}_{3} \mathrm{~L}$ was prepared by the literature methods. ${ }^{1}$ BIBP and BPY ligand was prepared on the basis of palladium-catalyzed cross-coupling reactions. IR absorption spectra of the compounds 1 - 3 were recorded in the range of 400-4000 $\mathrm{cm}^{-1}$ on a Nicolet (Impact 410) spectrometer with $\mathrm{KBr}$ pellets (5 mg of sample in 500 $\mathrm{mg}$ of $\mathrm{KBr}$ ). C, $\mathrm{H}$ and $\mathrm{N}$ analyses were carried out with a Perkin Elmer 240C elemental analyzer. Powder X-ray diffraction (PXRD) measurements were performed 
on a Bruker D8 Advance X-ray diffractometer using Mo- $\mathrm{K}_{\alpha}$ radiation $(\lambda=0.71073$ $\AA$ ), in which the X-ray tube was operated at $40 \mathrm{kV}$ and $40 \mathrm{~mA}$. The as-synthesized samples were characterized by thermogravimetric analysis (TGA) on a Perkin Elmer thermogravimetric analyzer Pyris 1 TGA up to $1023 \mathrm{~K}$ using a heating rate of $10 \mathrm{~K}$ $\min ^{-1}$ under $\mathrm{N}_{2}$ atmosphere.

Synthesis of complex 1. A mixture of DMF/ $\mathrm{H}_{2} \mathrm{O}(4 \mathrm{ml}$ : $0.5 / 3.5)$ containing the $\mathrm{H}_{3} \mathrm{~L}$ $(11.76 \mathrm{mg}, 0.03 \mathrm{mmol})$ and $\mathrm{Co}\left(\mathrm{NO}_{3}\right)_{2} \cdot 6 \mathrm{H}_{2} \mathrm{O}(8.73 \mathrm{mg}, 0.03 \mathrm{mmol})$ was mixed in a Teflon vessel within the autoclave. The vessel was heated at $95{ }^{\circ} \mathrm{C}$ for $72 \mathrm{~h}$ and then cooled to room temperature. Large quantities of red crystals were obtained and crystals were filtered off, washed with mother liquid, and dried under ambient conditions. The red crystals of 1 were collected in $40 \%$ yield (based on $\mathrm{H}_{3} \mathrm{~L}$ ligand). Elemental analysis calcd. for $\mathrm{C}_{44} \mathrm{H}_{30} \mathrm{CoO}_{14}$ (1): C, 62.79\%; H, 3.59\%; Found: $\mathrm{C}$, $62.80 \% ; \mathrm{H}, 3.71 \%$. The IR spectra of complex 1 is shown in the Figure S11.

Synthesis of complex 2. A mixture of $\mathrm{DMF} / \mathrm{H}_{2} \mathrm{O}\left(6 \mathrm{ml}\right.$ : 1/5) containing the $\mathrm{H}_{3} \mathrm{~L}$ (11.76 mg, $0.03 \mathrm{mmol}$ ), 4,4'-di(1H-imidazol-1-yl)-1,1'-biphenyl (BIBP) (8.60mg, $0.03 \mathrm{mmol})$ and $\mathrm{Co}\left(\mathrm{NO}_{3}\right)_{2} \cdot 6 \mathrm{H}_{2} \mathrm{O}(29.1 \mathrm{mg}, 0.1 \mathrm{mmol})$ was mixed in a Teflon vessel within the autoclave. The vessel was heated at $95{ }^{\circ} \mathrm{C}$ for $72 \mathrm{~h}$ and then cooled to room temperature. Large quantities of brownish red crystals were obtained and crystals were filtered off, washed with mother liquid, and dried under ambient conditions. The red crystals of 2 were collected in $37 \%$ yield (based on $\mathrm{H}_{3} \mathrm{~L}$ ligand). Elemental analysis calcd. for $\mathrm{C}_{40} \mathrm{H}_{30} \mathrm{CoN}_{4} \mathrm{O}_{8}(2)$ : $\mathrm{C}, 63.75 \% ; \mathrm{H}, 4.01 \%$; N, 7.43\%, Found: $\mathrm{C}$, $63.86 \%$; H, 4.11\%; N, 7.33\%; The IR spectra of complex 2 is shown in the Figure $\mathrm{S} 12$.

Synthesis of complex 3. A mixture of $\mathrm{DMF} / \mathrm{H}_{2} \mathrm{O}\left(6 \mathrm{ml}\right.$ : 1.5/4.5) containing the $\mathrm{H}_{3} \mathrm{~L}$ (11.76 mg, $0.03 \mathrm{mmol}), 4,4$ '-bipyridine (BPY) (7.00 $\mathrm{mg}, 0.05 \mathrm{mmol}$ ) and $\mathrm{Co}\left(\mathrm{NO}_{3}\right)_{2} \cdot 6 \mathrm{H}_{2} \mathrm{O}(8.73 \mathrm{mg}, 0.03 \mathrm{mmol})$ was mixed in a Teflon vessel within the autoclave. The vessel was heated at $95{ }^{\circ} \mathrm{C}$ for $72 \mathrm{~h}$ and then cooled to room temperature. Large quantities of orange red crystals were obtained and crystals were filtered off, washed with mother liquid, and dried under ambient conditions. The red crystals of 1 were collected in $48 \%$ yield (based on $\mathrm{H}_{3} \mathrm{~L}$ ligand). Elemental analysis calcd. for $\mathrm{C}_{74} \mathrm{H}_{54} \mathrm{Co}_{3} \mathrm{~N}_{6} \mathrm{O}_{16}$ (3): C, 60.87\%; H, 3.73\%; N, 5.76\%, Found: C, 60.89\%; H, 3.68\%; N, 5.78\%;. The IR spectra of complex 3 is shown in the Figure S13. 
X-ray crystallography. Single crystals of complexes 1-3 were tested on a Bruker SMART APEX CCD diffractometer using graphite monochromated Mo K $\alpha$ radiation $(\lambda=0.71073 \AA)$ at $296 \mathrm{~K}$. From the data reduction to the structure determination, the follow procedures were used: the International Tables for X-ray Crystallography, ${ }^{2}$ SAINT, ${ }^{3}$ SADABS, ${ }^{4}$ XPREP, ${ }^{5}$ SHELXTL-97 ${ }^{6}$ and SHELXTL-97 program $^{7}$ package.

Table S1. Crystal data and structural refinement parameters of complexes 1-3.

\begin{tabular}{|c|c|c|c|}
\hline Complexes & 1 & 2 & 3 \\
\hline Empirical formula & $\mathrm{C}_{44} \mathrm{H}_{30} \mathrm{CoO}_{14}$ & $\mathrm{C}_{40} \mathrm{H}_{30} \mathrm{CoN}_{4} \mathrm{O}_{8}$ & $\mathrm{C}_{74} \mathrm{H}_{54} \mathrm{Co}_{3} \mathrm{~N}_{6} \mathrm{O}_{16}$ \\
\hline Formula weight & 841.61 & 753.61 & 1460.02 \\
\hline Crystal system & Orthorhombic & Triclinic & Triclinic \\
\hline Space group & Pccn & $P \overline{\mathbf{1}}$ & $P \overline{\mathbf{1}}$ \\
\hline$a / \AA$ & $9.3781(18)$ & $9.0318(13)$ & $11.4194(5)$ \\
\hline$b / \AA$ & $18.800(4)$ & $14.344(2)$ & $11.5003(4)$ \\
\hline$c / \AA$ & $21.954(5)$ & $14.923(2)$ & $12.9926(5)$ \\
\hline$\alpha /{ }^{\circ}$ & 90 & $116.811(2)$ & $69.9180(10)$ \\
\hline$\beta /{ }^{\circ}$ & 90 & $94.012(2)$ & $81.2550(10)$ \\
\hline$\gamma /{ }^{\circ}$ & 90 & $98.805(2)$ & $87.5690(10)$ \\
\hline$V / \AA^{3}$ & $3870.7(14)$ & $1683.8(4)$ & $1583.82(11)$ \\
\hline$Z$ & 4 & 2 & 1 \\
\hline$D_{\text {calcd }} / \mathrm{g} \mathrm{cm}^{-3}$ & 1.444 & 1.486 & 1.531 \\
\hline$\mu / \mathrm{mm}^{-1}$ & 0.516 & 0.573 & 0.856 \\
\hline$F(000)$ & 1732 & 778 & 749 \\
\hline$\theta \min -\max /^{\circ}$ & $2.15,28.39$ & $1.55,25.50$ & $2.58,25.00$ \\
\hline Tot., uniq. data & 29664,4833 & 12945,6165 & 11390,5539 \\
\hline$R($ int $)$ & 0.0533 & 0.0434 & 0.0231 \\
\hline Nref, Npar & 4833,270 & 6165,480 & 5539,449 \\
\hline
\end{tabular}




\begin{tabular}{|c|c|c|c|}
\hline$R_{1}, w R_{2}[I>2 \sigma(I)]$ & $0.0397,0.0941$ & $0.0427,0.1305$ & $0.0502,0.1380$ \\
\hline GOF on $F^{2}$ & 1.069 & 1.110 & 1.108 \\
\hline Min. and max resd dens $\left(\mathrm{e} \cdot \AA^{-3}\right)$ & $0.384,-0.416$ & $0.540,-0.493$ & $1.161,-1.204$ \\
\hline
\end{tabular}

Table S2. Selected bond lengths ( $\AA$ ) and angles (deg) for complexes 1-3.

\begin{tabular}{|c|c|c|c|}
\hline \multicolumn{4}{|c|}{ Complex 1} \\
\hline $\mathrm{Co}(1)-\mathrm{O}(3) \# 3$ & $2.0686(12)$ & $\mathrm{Co}(1)-\mathrm{O}(3) \# 4$ & $2.0686(12)$ \\
\hline $\mathrm{Co}(1)-\mathrm{O}(6) \# 5$ & $2.1114(11)$ & $\mathrm{Co}(1)-\mathrm{O}(6) \# 1$ & $2.1114(12)$ \\
\hline $\mathrm{Co}(1)-\mathrm{O}(1) \# 6$ & $2.1233(12)$ & $\mathrm{Co}(1)-\mathrm{O}(1)$ & $2.1233(12)$ \\
\hline $\mathrm{O}(3)-\mathrm{Co}(1) \# 2$ & $2.0686(12)$ & $\mathrm{O}(3) \# 3-\mathrm{Co}(1)-\mathrm{O}(3) \# 4$ & $93.42(7)$ \\
\hline $\mathrm{O}(3) \# 3-\mathrm{Co}(1)-\mathrm{O}(6) \# 5$ & $89.72(5)$ & $\mathrm{O}(3) \# 4-\mathrm{Co}(1)-\mathrm{O}(6) \# 5$ & $99.70(5)$ \\
\hline $\mathrm{O}(3) \# 3-\mathrm{Co}(1)-\mathrm{O}(6) \# 1$ & $99.70(5)$ & $\mathrm{O}(3) \# 4-\mathrm{Co}(1)-\mathrm{O}(6) \# 1$ & $89.72(5)$ \\
\hline $\mathrm{O}(6) \# 5-\mathrm{Co}(1)-\mathrm{O}(6) \# 1$ & $166.30(7)$ & $\mathrm{O}(3) \# 3-\mathrm{Co}(1)-\mathrm{O}(1) \# 6$ & $171.24(5)$ \\
\hline $\mathrm{O}(3) \# 4-\mathrm{Co}(1)-\mathrm{O}(1) \# 6$ & $91.31(5)$ & $\mathrm{O}(6) \# 5-\mathrm{Co}(1)-\mathrm{O}(1) \# 6$ & $82.21(5)$ \\
\hline $\mathrm{O}(6) \# 1-\mathrm{Co}(1)-\mathrm{O}(1) \# 6$ & $87.68(5)$ & $\mathrm{O}(3) \# 3-\mathrm{Co}(1)-\mathrm{O}(1)$ & $91.31(5)$ \\
\hline $\mathrm{O}(3) \# 4-\mathrm{Co}(1)-\mathrm{O}(1)$ & $171.24(5)$ & $\mathrm{O}(6) \# 5-\mathrm{Co}(1)-\mathrm{O}(1)$ & $87.68(5)$ \\
\hline $\mathrm{O}(6) \# 1-\mathrm{Co}(1)-\mathrm{O}(1)$ & $82.21(5)$ & $\mathrm{O}(1) \# 6-\mathrm{Co}(1)-\mathrm{O}(1)$ & $84.98(7)$ \\
\hline \multicolumn{4}{|c|}{ Complex 2} \\
\hline $\mathrm{Co}(1)-\mathrm{O}(6) \# 1$ & $2.0358(18)$ & $\mathrm{Co}(1)-\mathrm{O}(5) \# 2$ & $2.0662(16)$ \\
\hline $\mathrm{Co}(1)-\mathrm{N}(1)$ & $2.132(2)$ & $\mathrm{Co}(1)-\mathrm{O}(1)$ & $2.1512(18)$ \\
\hline $\mathrm{Co}(1)-\mathrm{N}(2) \# 3$ & $2.172(2)$ & $\mathrm{Co}(1)-\mathrm{O}(2)$ & $2.2783(17)$ \\
\hline $\mathrm{O}(5)-\mathrm{Co}(1) \# 4$ & $2.0662(16)$ & $\mathrm{O}(6)-\mathrm{Co}(1) \# 1$ & $2.0358(18)$ \\
\hline $\mathrm{N}(2)-\mathrm{Co}(1) \# 5$ & $2.1720(19)$ & $\mathrm{O}(6) \# 1-\mathrm{Co}(1)-\mathrm{O}(5) \# 2$ & $97.83(7)$ \\
\hline $\mathrm{O}(6) \# 1-\mathrm{Co}(1)-\mathrm{N}(1)$ & $93.58(8)$ & $\mathrm{O}(5) \# 2-\mathrm{Co}(1)-\mathrm{N}(1)$ & $90.31(7)$ \\
\hline $\mathrm{O}(6) \# 1-\mathrm{Co}(1)-\mathrm{O}(1)$ & $153.78(7)$ & $\mathrm{O}(5) \# 2-\mathrm{Co}(1)-\mathrm{O}(1)$ & $108.35(7)$ \\
\hline
\end{tabular}




\begin{tabular}{|c|c|c|c|}
\hline $\mathrm{N}(1)-\mathrm{Co}(1)-\mathrm{O}(1)$ & $85.01(8)$ & $\mathrm{O}(6) \# 1-\mathrm{Co}(1)-\mathrm{N}(2) \# 3$ & $95.51(8)$ \\
\hline $\mathrm{O}(5) \# 2-\mathrm{Co}(1)-\mathrm{N}(2) \# 3$ & $86.56(7)$ & $\mathrm{N}(1)-\mathrm{Co}(1)-\mathrm{N}(2) \# 3$ & $170.71(8)$ \\
\hline $\mathrm{O}(1)-\mathrm{Co}(1)-\mathrm{N}(2) \# 3$ & $87.68(8)$ & $\mathrm{O}(6) \# 1-\mathrm{Co}(1)-\mathrm{O}(2)$ & $95.16(7)$ \\
\hline $\mathrm{O}(5) \# 2-\mathrm{Co}(1)-\mathrm{O}(2)$ & $165.89(7)$ & $\mathrm{N}(1)-\mathrm{Co}(1)-\mathrm{O}(2)$ & $94.39(7)$ \\
\hline $\mathrm{O}(1)-\mathrm{Co}(1)-\mathrm{O}(2)$ & $58.97(7)$ & $\mathrm{N}(2) \# 3-\mathrm{Co}(1)-\mathrm{O}(2)$ & $86.71(7)$ \\
\hline \multicolumn{4}{|c|}{ Complex 3} \\
\hline $\mathrm{N}(3)-\mathrm{Co}(1) \# 1$ & $2.177(3)$ & $\mathrm{Co}(1)-\mathrm{O}(2) \# 2$ & $2.023(2)$ \\
\hline $\mathrm{Co}(1)-\mathrm{O}(1)$ & $2.027(2)$ & $\mathrm{Co}(1)-\mathrm{O}(3) \# 3$ & $2.102(2)$ \\
\hline $\mathrm{Co}(1)-\mathrm{N}(2)$ & $2.151(3)$ & $\mathrm{Co}(1)-\mathrm{N}(3) \# 4$ & $2.177(3)$ \\
\hline $\mathrm{Co}(1)-\mathrm{O}(4) \# 3$ & $2.308(3)$ & $\mathrm{Co}(3)-\mathrm{O}(7) \# 5$ & $2.050(4)$ \\
\hline $\mathrm{Co}(3)-\mathrm{O}(7)$ & $2.050(4)$ & $\mathrm{Co}(3)-\mathrm{O}(6)$ & $2.168(3)$ \\
\hline $\mathrm{Co}(3)-\mathrm{O}(6) \# 5$ & $2.168(3)$ & $\mathrm{Co}(3)-\mathrm{N}(1)$ & $2.191(5)$ \\
\hline $\mathrm{Co}(3)-\mathrm{N}(1) \# 5$ & $2.191(5)$ & $\mathrm{O}(2)-\mathrm{Co}(1) \# 2$ & $2.023(2)$ \\
\hline $\mathrm{O}(4)-\mathrm{Co}(1) \# 3$ & $2.308(3)$ & $\mathrm{O}(3)-\mathrm{Co}(1) \# 3$ & $2.102(2)$ \\
\hline $\mathrm{O}(2) \# 2-\mathrm{Co}(1)-\mathrm{O}(1)$ & $110.77(10)$ & $\mathrm{O}(2) \# 2-\mathrm{Co}(1)-\mathrm{O}(3) \# 3$ & $156.16(10)$ \\
\hline $\mathrm{O}(1)-\mathrm{Co}(1)-\mathrm{O}(3) \# 3$ & $92.22(10)$ & $\mathrm{O}(2) \# 2-\mathrm{Co}(1)-\mathrm{N}(2)$ & $91.26(10)$ \\
\hline $\mathrm{O}(1)-\mathrm{Co}(1)-\mathrm{N}(2)$ & $92.19(11)$ & $\mathrm{O}(3) \# 3-\mathrm{Co}(1)-\mathrm{N}(2)$ & $94.22(11)$ \\
\hline $\mathrm{O}(2) \# 2-\mathrm{Co}(1)-\mathrm{N}(3) \# 4$ & $85.22(10)$ & $\mathrm{O}(1)-\mathrm{Co}(1)-\mathrm{N}(3) \# 4$ & $90.38(11)$ \\
\hline $\mathrm{O}(3) \# 3-\mathrm{Co}(1)-\mathrm{N}(3) \# 4$ & $88.46(11)$ & $\mathrm{N}(2)-\mathrm{Co}(1)-\mathrm{N}(3) \# 4$ & $176.21(11)$ \\
\hline $\mathrm{O}(2) \# 2-\mathrm{Co}(1)-\mathrm{O}(4) \# 3$ & $97.44(9)$ & $\mathrm{O}(1)-\mathrm{Co}(1)-\mathrm{O}(4) \# 3$ & $150.75(10)$ \\
\hline $\mathrm{O}(3) \# 3-\mathrm{Co}(1)-\mathrm{O}(4) \# 3$ & $59.00(9)$ & $\mathrm{N}(2)-\mathrm{Co}(1)-\mathrm{O}(4) \# 3$ & $94.89(11)$ \\
\hline $\mathrm{N}(3) \# 4-\mathrm{Co}(1)-\mathrm{O}(4) \# 3$ & $84.19(11)$ & $\mathrm{O}(7) \# 5-\mathrm{Co}(3)-\mathrm{O}(7)$ & $180.00(15)$ \\
\hline $\mathrm{O}(7) \# 5-\mathrm{Co}(3)-\mathrm{O}(6)$ & $117.37(12)$ & $\mathrm{O}(7)-\mathrm{Co}(3)-\mathrm{O}(6)$ & $62.63(12)$ \\
\hline $\mathrm{O}(7) \# 5-\mathrm{Co}(3)-\mathrm{O}(6) \# 5$ & $62.63(12)$ & $\mathrm{O}(7)-\mathrm{Co}(3)-\mathrm{O}(6) \# 5$ & $117.37(12)$ \\
\hline $\mathrm{O}(6)-\mathrm{Co}(3)-\mathrm{O}(6) \# 5$ & $180.000(1)$ & $\mathrm{O}(7) \# 5-\mathrm{Co}(3)-\mathrm{N}(1)$ & $88.53(19)$ \\
\hline
\end{tabular}




\begin{tabular}{|l|l|l|l|}
\hline $\mathrm{O}(7)-\mathrm{Co}(3)-\mathrm{N}(1)$ & $91.47(19)$ & $\mathrm{O}(6)-\mathrm{Co}(3)-\mathrm{N}(1)$ & $90.61(14)$ \\
\hline $\mathrm{O}(6) \# 5-\mathrm{Co}(3)-\mathrm{N}(1)$ & $89.39(14)$ & $\mathrm{O}(7) \# 5-\mathrm{Co}(3)-\mathrm{N}(1) \# 5$ & $91.47(19)$ \\
\hline $\mathrm{O}(7)-\mathrm{Co}(3)-\mathrm{N}(1) \# 5$ & $88.53(19)$ & $\mathrm{O}(6)-\mathrm{Co}(3)-\mathrm{N}(1) \# 5$ & $89.39(14)$ \\
\hline $\mathrm{O}(6) \# 5-\mathrm{Co}(3)-\mathrm{N}(1) \# 5$ & $90.61(14)$ & $\mathrm{N}(1)-\mathrm{Co}(3)-\mathrm{N}(1) \# 5$ & $180.00(2)$ \\
\hline
\end{tabular}

Symmetry Codes: for $1: \# 1=-x+2,-y+1,-z+1, \# 2=x-1,-y+3 / 2, z+1 / 2, \# 3=-x+1 / 2, y, z-$ 1/2, \#4 = x +1, $-y+3 / 2, z-1 / 2, \# 5=x-1 / 2, y+1 / 2,-z+1, \# 6=-x+3 / 2,-y+3 / 2$, z.; for 2: \#1 = -x

$+1,-y,-z, \# 2=x, y+1, z, \# 3=x-1, y, z+1, \# 4=x, y-1, z, \# 5=x+1, y, z-1$; for 3: \#1 = x - 1, y, z, $\# 2=-x+2,-y+2,-z+1, \# 3=-x+2,-y+1,-z+2, \# 4=x+1, y, z, \# 5=-x+1,-y+1,-z, \# 6=-$ $x+1,-y,-z$.

\section{Powder X-ray diffraction patterns of complexes 1-3.}

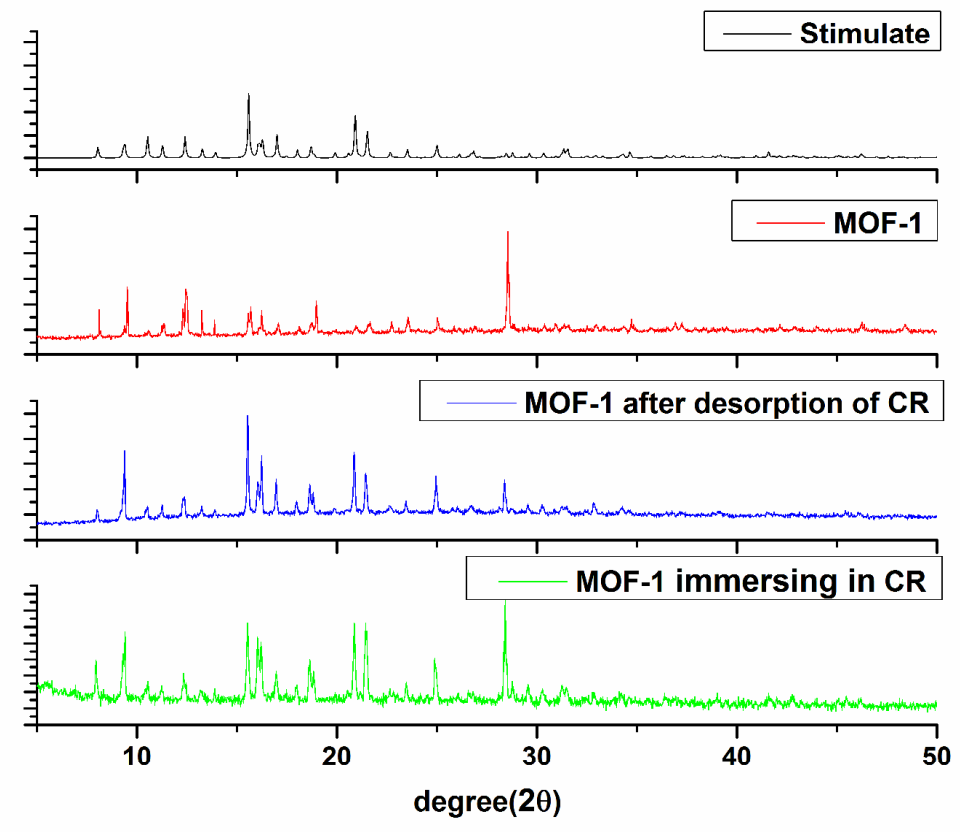

Figure S1 Powder X-ray diffraction patterns of complex 1. 


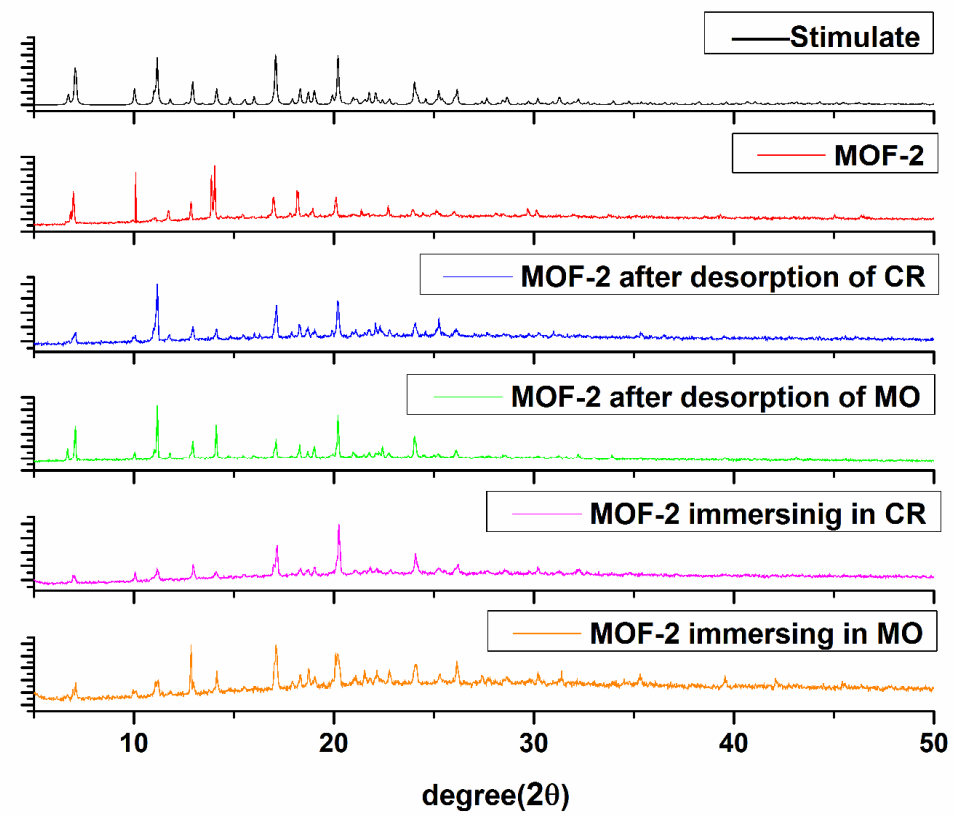

Figure S2 Powder X-ray diffraction patterns of complex 2.
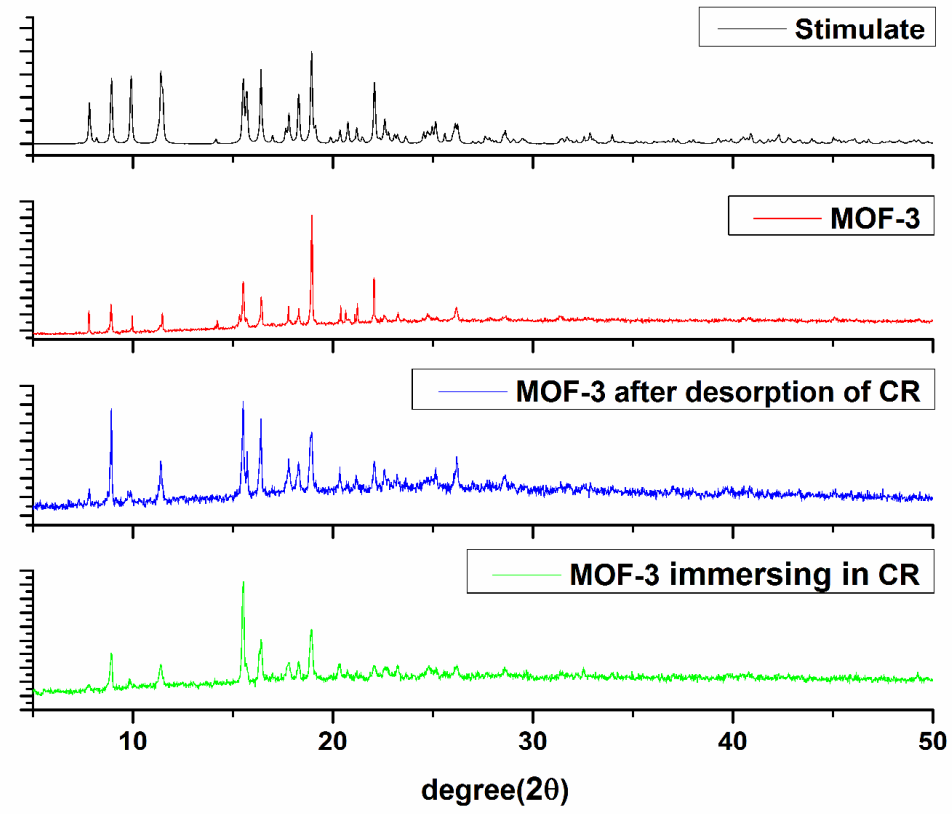

Figure S3 Powder X-ray diffraction patterns of complex 3. 


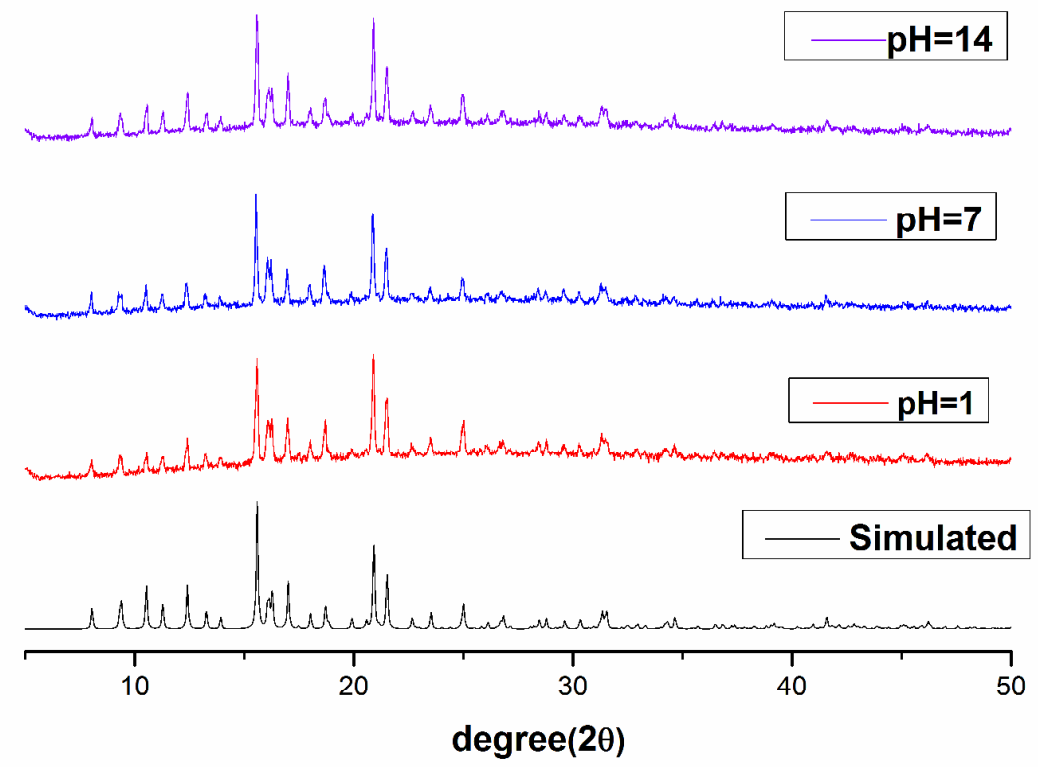

Figure S4 Powder X-ray diffraction patterns of complex 1 after being soaked in water, acidic and basic solutions for $12 \mathrm{~h}$.
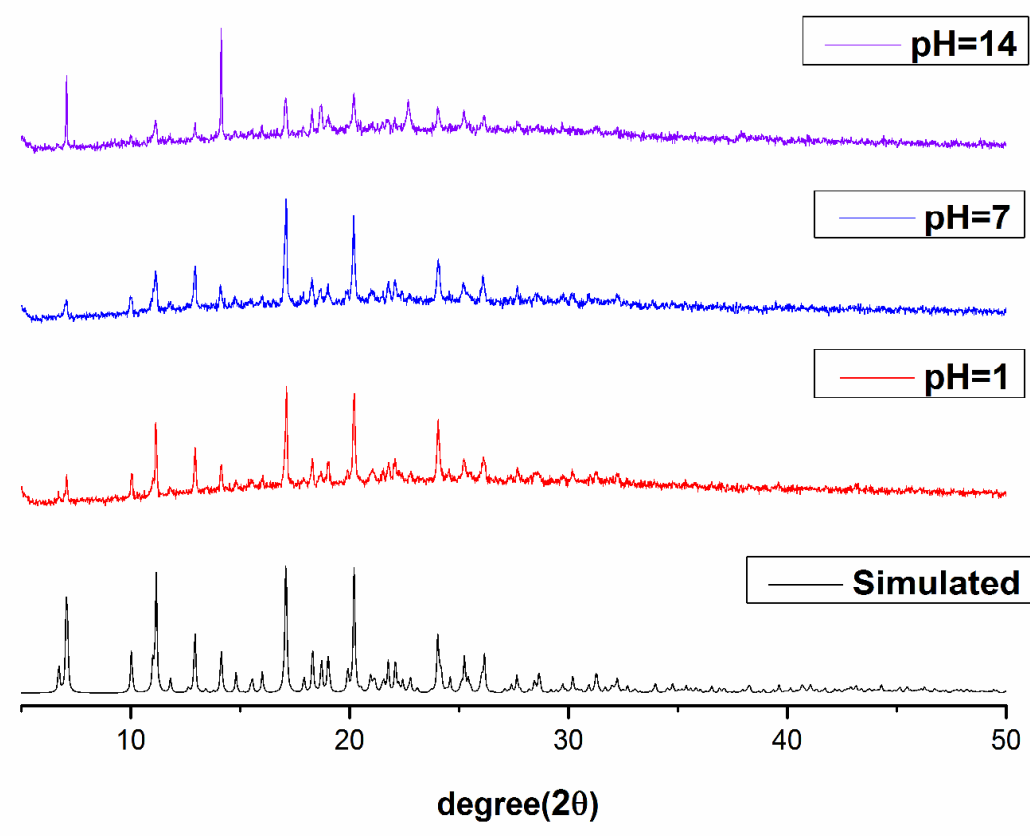

Figure S5 Powder X-ray diffraction patterns of complex 2 after being soaked in water, acidic and basic solutions for $12 \mathrm{~h}$. 


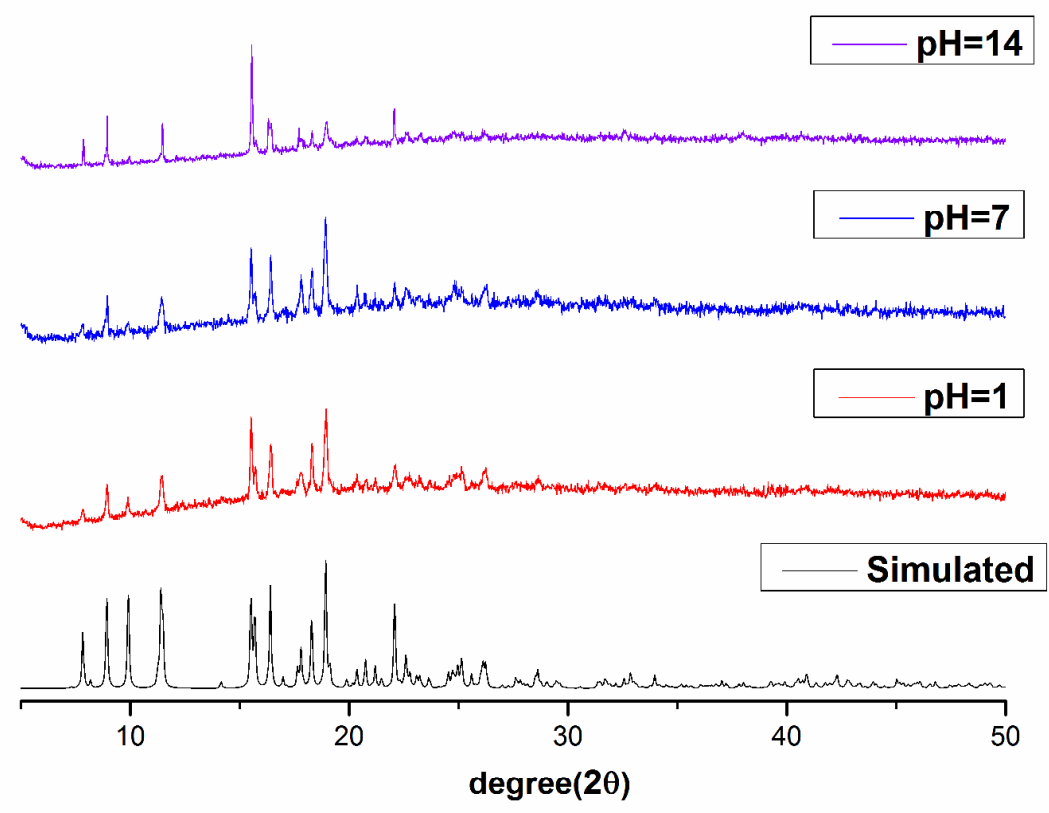

Figure S6 Powder X-ray diffraction patterns of complex 3 after being soaked in water, acidic and basic solutions for $12 \mathrm{~h}$. 


\section{Photographic images}

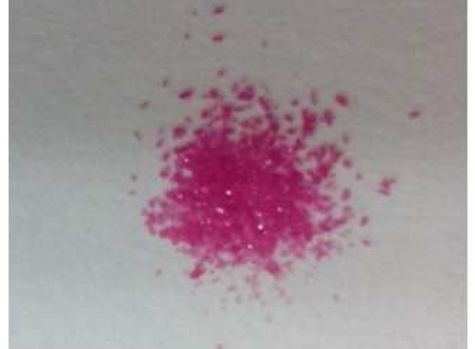

MOF-1 before absorption

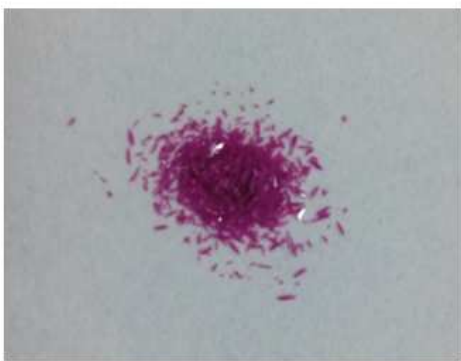

MOF-2 before absorption

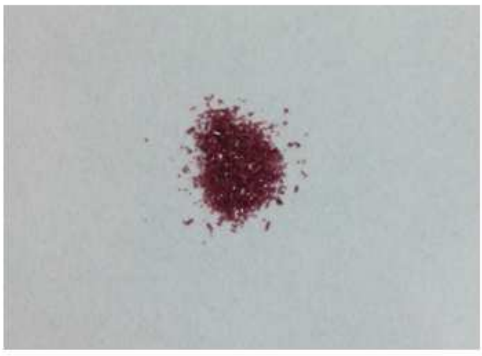

MOF-3 before absorption

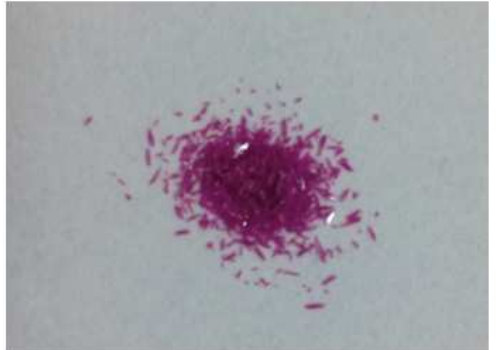

MOF-2 before absorption

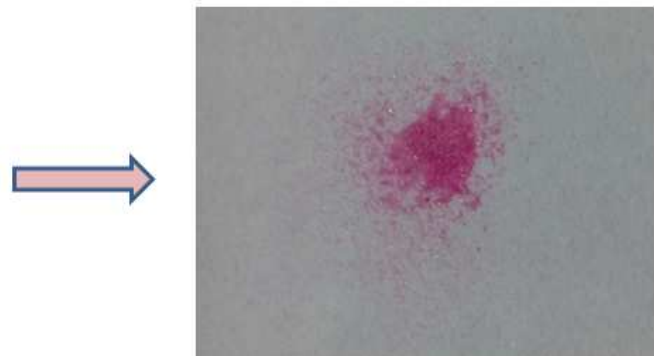

MOF-1 after absorption of CR

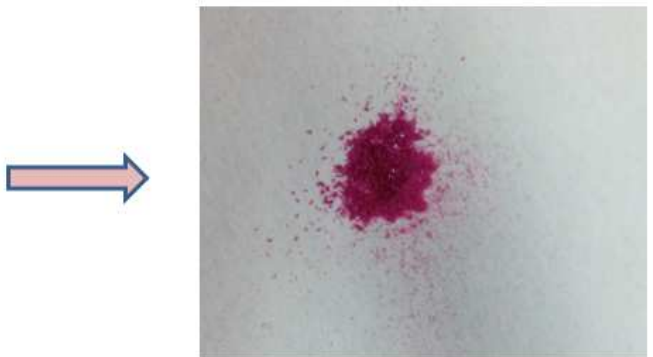

MOF-2 after absorption of CR

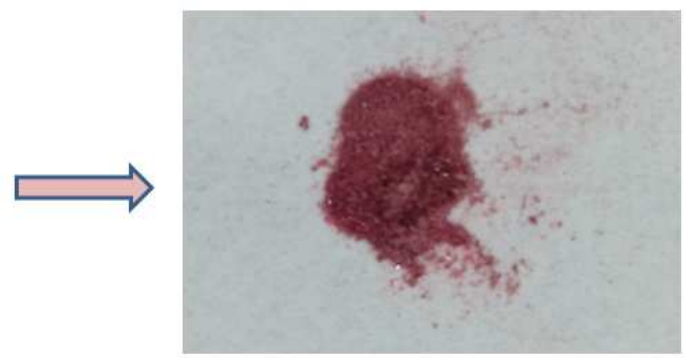

MOF-3 after absorption of CR

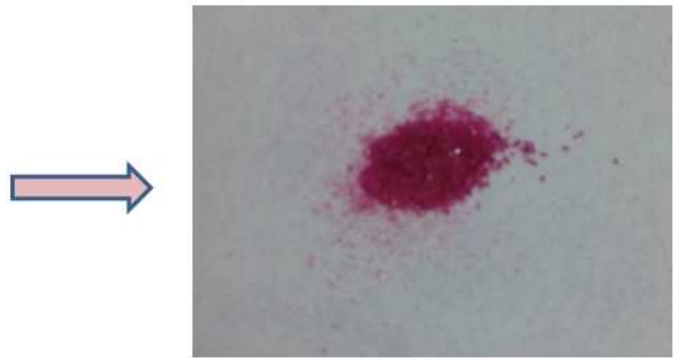

MOF-2 after absorption of MO

Figure S7. Photographic images of MOFs before and after absorption of anionic dyes. 
4. IR spectra of complexes 1-3 before and after the absorption of dyes and related ligands.

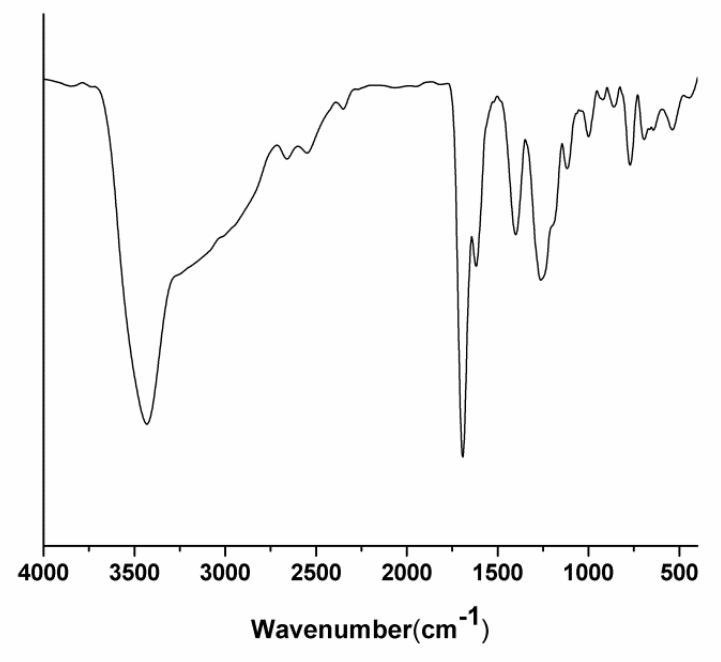

Figure S8 IR spectra of $\mathrm{H}_{3} \mathrm{~L}$ ligand.

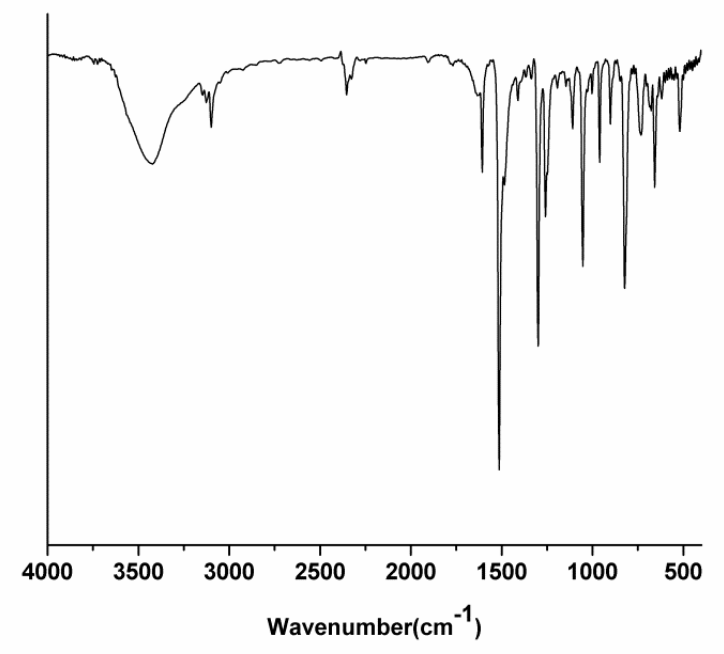

Figure S9 IR spectra of BIBP ligand. 


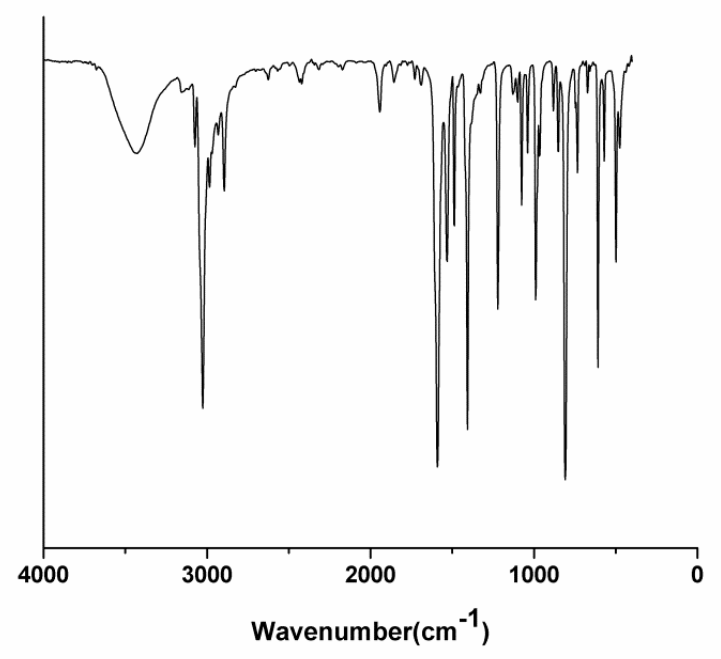

Figure S10 IR spectra of BPY ligand.

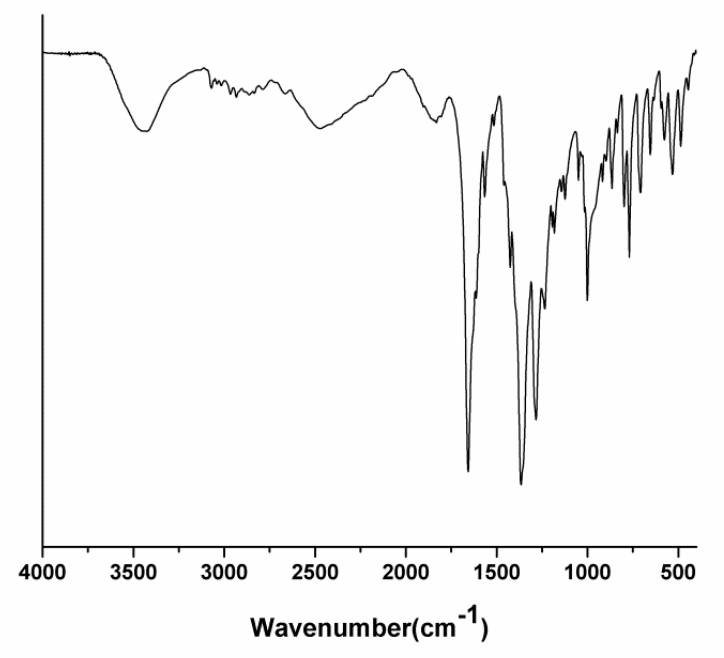

Figure S11 IR spectra of complex 1. 


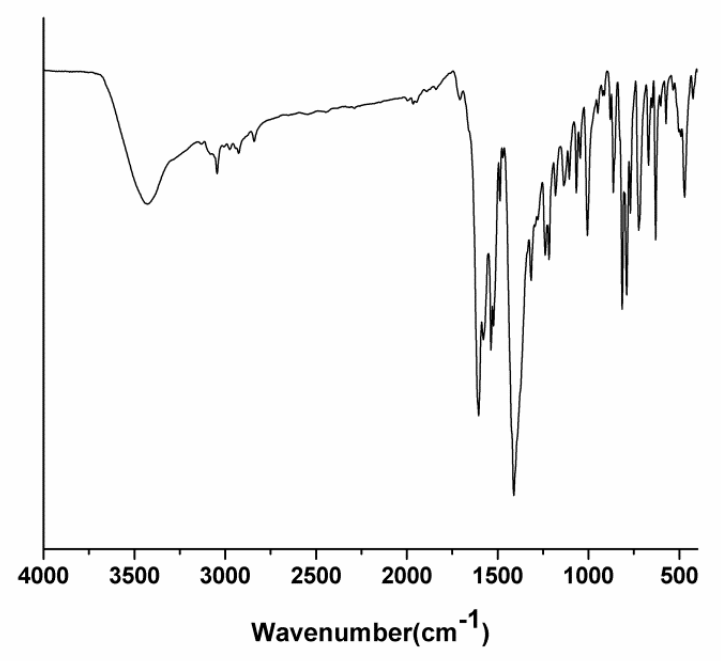

Figure S12 IR spectra of complex 2.

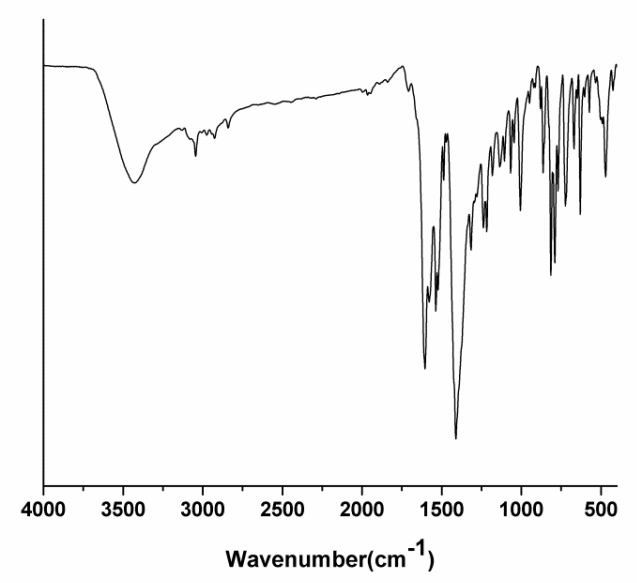

Figure S13 IR spectra of complex 3 .

\section{The TGA diagrams of complexes $1-3$.}

To characterize the stability of the three complexes, thermogravimetric analyses (TGA) (Figure S14) were studied in detail. For complex 1, the TGA curve shows that it can keep stable to about $380{ }^{\circ} \mathrm{C}$, after this temperature, the structure starts to decompose. From 25 to $169^{\circ} \mathrm{C}$, the weightlessness $2.5 \%$ for complex 2 is equivalent 
of lossing one water molecule (calcd 2.4\%), then, the TGA curve is almost flat until $351{ }^{\circ} \mathrm{C}$, upon this temperature, TGA curve shows a rapid weight loss the complex begins to collapse. The skeleton of Complex 3 starts to decompose until $383{ }^{\circ} \mathrm{C}$.

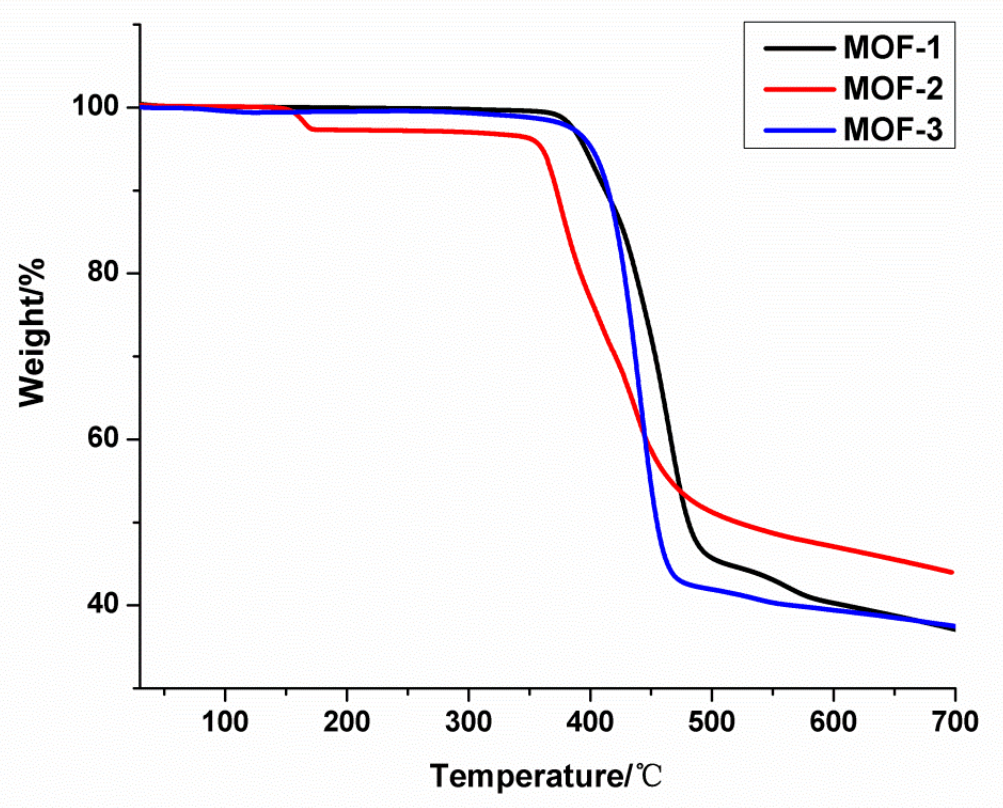

Figure S14 The TGA diagrams of complexes 1-3.

\section{The UV-visible spectra of complexes 1-3.}

Due to the different color of the three complexes, we test their solid state UV adsorption. The UV-vis absorption spectra of crystals 1-3 were tested in their crystalline state at room temperature (Figure S15). The spectra of three complexes show absorption band in the range of $200-420 \mathrm{~nm}$ (absorption maxima is $237 \mathrm{~nm}$ for BPY and $242 \mathrm{~nm}$ for BIBP, 320nm for $\mathrm{H}_{3} \mathrm{~L}$, respectively), which can be considered as $\pi-\pi^{*}$ transitions of the ligands. Another wide band from $430-625 \mathrm{~nm}$ can be ascribed to the spin-allowed $d-d$ electronic transitions of the $d^{7}\left(\mathrm{Co}^{2+}\right)$ cation. The different colors of three complexes result from the absorption band and absorption intensity. 


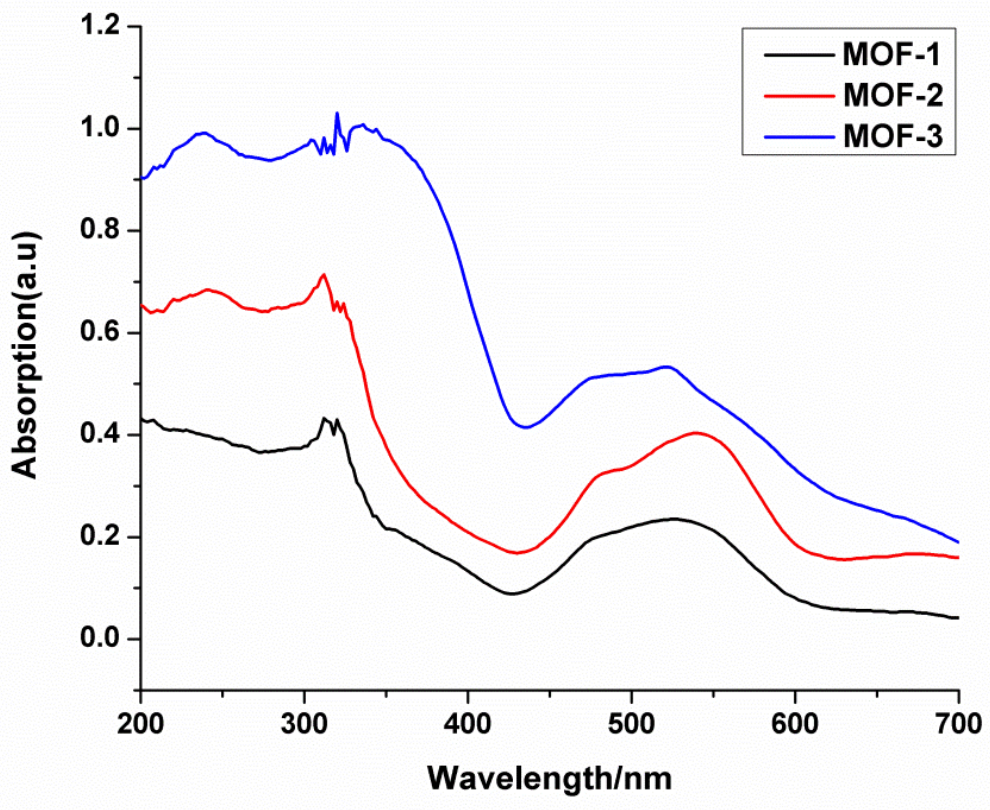

Figure S15 The UV-vis spectra of complexes 1-3. 


\section{References}

1. Schnobrich, J. K.; Lebel, O.; Cychosz, K. A.; Dailly, A.; Wong-Foy, A. G.; Matzger, A. J. J. Am. Chem. Soc. 2010,132, 13941.

2. International Tables for X-Ray Crystallography; Kynoch Press:Birmingham, England, 1952; Vol. III.

3. SAINT, version 6.02; Bruker AXS: Madison, WI, 1999.

4. Sheldrick, G. M. SADABS: Empirical Absorption Correction-Program; University of Göttingen: Göttingen, Germany, 1997.

5. XPREP, version 5.1; Siemens Industrial Automation Inc.: Madison, WI, 1995.

6. Sheldrick, G. M. SHELXTL Reference Manual, version 5.1; Bruker AXS: Madison, WI, 1997.

7. Sheldrick, G. M. SHELXL-97: Program for Crystal Structure Refinement; University of Gottingen: Gottingen, Germany, 1997. 